\title{
Invariants of 3-Manifolds Associated with Quantum Groups and Verlinde's Formula
}

By

\author{
Toshie TAKATA*
}

\begin{abstract}
We obtain a projectively linear representation of $S L(2, Z)$ from invariants defined by Reshetikhin and Turaev and prove 'Verlinde's formula' for $S U(2)$ based on the computation of invariants. Using an algebra associated with 'Ising model', we constract invariants of links and 3 -manifolds.
\end{abstract}

\section{Introduction}

In [16], Witten obtained new topological invariants of closed 3-manifolds and links in 3-manifolds from the quantum field theory. Shortly afterwards, in [13], Reshetikhin and Turaev defined related invariants of closed oriented 3-manifolds and links in such 3-manifolds, by means of representations of quantum groups. More precisely, they use quantized universal enveloping algebra $U_{q}(\operatorname{sl}(2, \boldsymbol{C}))$, which is a $q$-deformation of the universal enveloping algebra of $\operatorname{sl}(2, C)$ discovered independently by Drinfeld [1] and Jimbo ([3], [4]). The algebra $U_{q}(s l(2, \boldsymbol{C}))$ has a structure of a Hopf algebra. Reshetikhin and Turaev introduced the additional structure in the case $q=\exp (2 m \pi \sqrt{-1} / r)$ called a 'modular' Hopf algebra to define invariants of 3-manifolds. They obtain invariants of 3-manifolds as a combinational formula using invariants of framed link associated with the algebra $U_{q}(s l(2, C))$. This is based on the fact that any closed connected oriented 3-manifold is obtained by Dehn surgery [12] of $S^{3}$ along a framed link [9].

As an application of the invariants, we construct a projectively linear representation of $S L(2, Z)$. Let $Z\left(T^{2}\right)$ be an $(r-1)$-dimensional vector space over $C$ and $\left\{e_{i}\right\}_{i=0}^{r-2}$ a basis of the vector space $Z\left(T^{2}\right)$ and we associate to a basis element $e_{i}$ a solid torus $U_{i}$ which has a link in the interior. Gluing such two solid tori $U_{i}$ and $U_{3}$ by an element $X$ of the mapping class group of the torus $T^{2}$, we obtain a closed 3 -manifold $M_{X}$ with a link. We denote the invariant of the resulting manifold $M_{X}$ by $X_{i j}$. We define an action $\rho$ of $S L(2, \boldsymbol{Z})$

Communicated by M. Kashiwara, April 12, 1991. Revised June 17, 1991.

1991 Mathematics Subject Classifications : 57M25, 81R50.

* Department of Mathematics, Kyushu University 33, Fukuoka 812, Japan. 
on the vector space $Z\left(T^{2}\right)$ by the formula

$$
\rho(X) e_{\jmath}=\sum_{\imath=0}^{r-2} X_{i j} e_{\imath} \quad(j=0, \cdots, r-2) .
$$

For generators $S$ and $T$ of $S L(2, Z \mathbb{Z})$, we obtain the equations

$$
\begin{aligned}
& S_{i J}=\sqrt{\frac{2}{r}} \sin \frac{m(i+1)(j+1) \pi}{r}, \\
& T_{i \jmath}=q^{i(\imath+2) / 4} \delta_{i \jmath} .
\end{aligned}
$$

This matrix $\left(S_{i j}\right)$ is the unitary matrix and the representation of $S L(2, \mathbb{Z})$ by means of the matrices above was discovered by Kac and Peterson [5] to discribe the modular property of the character of the affine Lie algebra and was also used by Kohno [7] to define invariants of 3 -manifolds. The above representation

$$
\rho: S L(2, Z) \longrightarrow G L\left(Z\left(T^{2}\right)\right) /\langle C\rangle,
$$

where $\langle C\rangle$ is the cyclic group generated by a root of unity $C=$ $\exp (\sqrt{-1}(-\varphi+(3 \pi m / 2 r)-(\pi / 2)))$, is a projectively linear representation. Here $\varphi$ is determined from the following Gauss sum :

$$
\sqrt{2 r} \exp (\sqrt{-1} \varphi)=\sum_{k=0}^{2 r-1} \exp \left(\sqrt{-1} \pi k^{2} m / 2 r\right)
$$

As an application, we prove 'Verlinde's Formula' for $S U(2)$ [15]. This is given by the following formula:

$$
\frac{S_{i j} S_{i k}}{S_{i 0}}=\sum_{l=0}^{r-2} S_{i l} N_{l \jmath k}
$$

where

$$
N_{i J k}= \begin{cases}1 & \text { if }|i-j| \leqq k \leqq i+j, i+j+k \in 2 Z, i+-j+k \leqq 2(r-2) \\ 0 & \text { otherwise. }\end{cases}
$$

We verify it by computing the invariant of $S^{2} \times S^{1}$ with a link in two ways. The proof is similar to that by Witten [16], but our approach is based on representations of $U_{q}(s l(2, C))$ with $q=\exp (2 m \pi \sqrt{ } \overline{-1} / r)$.

Finally, instead of the above modular Hopf algebra, we consider an algebra associated with 'Ising model'. Recently, it has been discovered that this algebra is related for example to the conformal field theory (see for example [2], [10]), the representation theory of the infinite dimensional Lie algebras. This algebra is an associative algebra with 3 generators $1, \sigma, \phi$ whose relations are $\phi \cdot \phi=1$, $\phi \cdot \sigma=\sigma \cdot \psi=\sigma, \sigma \cdot \sigma=1+\phi$, and has the conformal dimensions $\Delta_{1}=0, \Delta_{\psi}=1 / 2$, $\Delta_{\sigma}=1 / 16$. Using this algebra, we define $\boldsymbol{C}$-linear operators for tangle diagrams and construct invariants of framed links. Then, by the same way as in [13], one can obtain invariants of closed oriented 3-manifolds. The topological invariance follows from the invariance under Kirby moves [6]. 
The paper is organized as follows. In $\S 1$, we review some of the results in [13]. We explain a representation of a modular Hopf algebra and define invariants of links and 3-manifolds derived by Reshetikhin and Turaev. In $\S 2$, using the invariants derived in $\S 1$, we establish a representation of $S L(2, \boldsymbol{Z})$. The action of generators $S$ and $T$ on the vector space $Z\left(T^{2}\right)$ is represented by matrices and it is shown that they satisfy their relations. In $\S 3$, a proof of 'Verlinde's formula' for $S U(2)$ is presented. To compute the invariants, we make use of the idea in $\S 2$. In $\S 4$, an algebra associated with 'Ising model' is described. Based on the algebra, we define invariants of framed links and obtain invariants of 3 -manifolds by means of the link invariants by a similar way as in $\S 1$.

\section{\$1. Review}

\subsection{Modular Hopf Algebra $U_{t}$}

In [13], Reshetikhin and Turaev give $U_{t}$ as an example of 'modular' Hopf algebra. In this paper, we consider the definition of topological invariants of 3-manifolds for this modular Hopf algebra $U_{t}$. We explain this modular Hopf algebra $U_{t}$. For a non zero $q \subseteq C, U_{q}(s l(2, C))$ is the Hopf algebra which is a $q$-deformation of the universal enveloping algebra of Lie algebra $\operatorname{sl}(2, \boldsymbol{C})$. Let us recall the definition of $U_{t}$ due to Reshetikhin and Turaev. Let $q$ be a root of unity and $t=\exp (\pi \sqrt{-1} m / 2 r)$ where $m$ and $r$ are mutually prime integers with odd $m, 2 r-1 \geqq m \geqq 1, r \geqq 2$ and $q=t^{4}$. We fix an integer $r$ satisfying $r \geqq 2$. We define $U_{t}$ to be the associative algebra with unit over the cyclotomic field $\boldsymbol{Q}(t)$ with 4 generators $K, K^{-1}, X, Y$ satisfying the following relations:

$$
\begin{aligned}
& X Y-Y^{Y} X=\frac{K^{2}-K^{-2}}{t^{2}-t^{-2}} \\
& X K=t^{-2} K X, Y K=t^{2} K Y \\
& K^{4 r}=1, X^{r}=Y^{r}=0 .
\end{aligned}
$$

The relations (1.1.1), (1.1.2) define the algebra $U_{q}(s l(2, C))$. The structure of Hopf algebra in $U_{q}(s l(2, C))$ induces a structure of a Hopf algebra in $U_{t}$. The action of comultiplication $\Delta$, counit $\varepsilon$, antipode $\gamma$ are given on the generators by the following formulas.

$$
\begin{aligned}
& \Delta(X)=X \otimes K+K^{-1} \otimes X \\
& \Delta(X)=Y \otimes K+K^{-1} \otimes I^{\prime} \\
& \Delta(K)=K \otimes K \\
& \varepsilon(X)=\varepsilon(Y)=0, \varepsilon(K)=1 \\
& \gamma(X)=-t^{2} X, \gamma(Y)=-t^{-2} Y, \gamma(K)=K^{-1} .
\end{aligned}
$$


The structure of the ribbon Hopf algebra in $U_{q}(s l(2, C))$ induces a structure of the ribbon Hopf algebra in $U_{t}$. Thus $U_{t}$ has the universal $R$-matrix $R \in$ $U_{t} \otimes U_{t}$ due to Drinfel'd [1] which satisfies Yang Baxter equation, $u \in U_{t}$ defined from $R$, and $v \in U_{t}$ which is a central element of $U_{t}$. If we write as sum $R=\sum_{i} \alpha_{i} \otimes \beta_{i}$, then $u=\sum_{i} \gamma\left(\beta_{i}\right) \alpha_{i}$ and $v=u K^{-2}$. Moreover, $U_{t}$ satisfies six axioms (see $[13, \S 3]$ ) and has a structure of modular Hopf algebra. We describe the representation of modular Hopf algebra $U_{t}$. Let $I$ be a finite set of integers $\{0,1, \cdots, r-2\}$. For an integer $i \in I, V_{\imath}$ denotes $(i+1)$-dimensional irreducible representation of $U_{t}$. It is an $(i+1)$-dimensional $U_{t}$-module. The action $\rho$ of the generator $K$ of $U_{t}$ on $V_{i}$ has the following matrix representation:

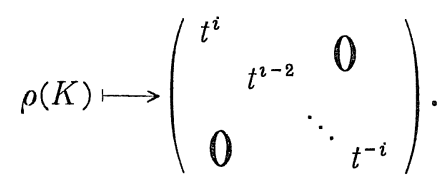

For any $U_{t}$-module $V_{i}$ we provide the dual linear space $V_{i}^{\vee}=\operatorname{Hom}_{C}(V, C)$ with the action of $U_{t}$ :

$$
\rho_{V_{i}}^{\vee}(a)=\left(\rho_{V_{i}}(\gamma(a))\right)^{*} \in \operatorname{End} V_{Y}^{\bigvee} \text { 。 }
$$

The matrix representation of this action is given by the following matrix :

$$
\rho_{V,} \vee(K) \longmapsto\left(\begin{array}{ccc}
t^{-i} & & 0 \\
& t^{-i+2} & 0 \\
0 & \ddots & t^{2}
\end{array}\right) .
$$

Let $V_{i}, V_{j}$ be $U_{t}$-modules and $\rho_{V_{i}}$ (resp. $\rho_{V_{j}}$ ) the action of $U_{t}$ on $V_{i}{ }_{i}$ (resp. $V_{\jmath}$ ). Their tensor product is the $U_{t}$-module $V_{i} \otimes V$, equipped with the action of $U_{t}$ defined by the formula for $a \in U_{t}$ :

$$
\rho_{V_{\imath} \otimes V_{j}}(a)=\left(\rho_{V_{\imath}} \otimes \rho_{V_{\jmath}}\right)(\Delta(a)) \text {. }
$$

Here $\Delta$ is the comultiplication of $U_{t}$. One may consider the category $\operatorname{Rep} U_{t}$ of finite dimensional linear representations of $U_{t}$. The objects of $R e p U_{t}$ are left $U_{t}$-modules

$$
V_{i l}^{\varepsilon_{l}} \otimes \cdots \otimes V_{i_{k}}^{\varepsilon_{k}}
$$

where $i_{l} \in I, \varepsilon_{l} \in\{ \pm 1\}, V_{i_{l}}^{+1}=V_{i_{l}}, V_{\imath_{l}}^{-1}=V_{\imath_{l}}^{\vee}, 1 \leqq l \leqq k$. The morphisms of RepUt are $U_{t}$-linear homomorphisms.

Definition 1.1. Let $V$ be an object of $\operatorname{Rep} U_{t}$. For any linear operator $f: V \rightarrow V$, we define its quantum trace $\operatorname{tr}_{q} f$ to be the ordinary trace over $\mathbb{C}$ of the linear operator

$$
f^{\prime}: V \longrightarrow V, f^{\prime}(x)=\rho\left(u^{-1} v\right) f(x)
$$


In particular, if $f$ is the identity map $i d_{V}$, then we denote $\operatorname{tr}_{q} i d_{V}$ by $\operatorname{dim}_{q} V$ and call it the quantum dimension of $V$. Note that if $V=V_{\jmath}$, for $j \in I$, then using $v=u K^{2}$ and (1.1.9), we get

$$
\begin{aligned}
\operatorname{dim}_{q} V_{j} & =\operatorname{tr}_{q}\left(i d_{V_{j}}\right)=\operatorname{Tr}\left(\rho_{V_{j}}\left(K^{2}\right) i d_{V_{j}}\right) \\
& =\sum_{n=0}^{j} t^{j-2 n}=\frac{t^{2 j+2}-t^{-2 j-2}}{t^{2}-t^{-2}}=[j+1]
\end{aligned}
$$

where $[n]=\left(t^{2 n}-t^{-2 n} / t^{2}-t^{-2}\right)=(\sin (\pi m n / r) / \sin (\pi m / r))$.

In [13], Reshetikhin and Turaev proved the following theorem.

Theorem 1.2 (Reshetikhin-Turaev). Let $V_{i}(i \in I)$ be an irreducible representation of $U_{t}$. There exists a decomposition

$$
V_{i} \otimes V_{j}=\left(\oplus_{k} V_{k}\right) \oplus Z_{i j}
$$

as a $U_{t}$-module, where $k$ satisfies the following conditions

$$
\begin{aligned}
& |i-j| \leqq k \leqq i+j, i+j+k \in 2 \boldsymbol{Z}, \\
& i+j+k \leqq 2(r-2) .
\end{aligned}
$$

Moreover $Z_{i}$ is a $U_{t}$-module with the following property. For any integers $i, j \in I$ and any $U_{t}$-linear homomorphism $f: Z_{i j} \rightarrow Z_{i j}$, the quantum trace of $f$ is equal to zero:

$$
\operatorname{tr}_{q} f=0
$$

\subsection{Ribbon Graph}

An oriented, directed, homogeneous ribbon tangle is a collection of ribbons and annuli as illustrated in Fig. 1 ([13], [14]).

A ribbon (annulus) is oriented if it has an orientation as a surface in $\boldsymbol{R}^{3}$. By the shaded regions, we express that the tangle is oriented (Fig. 1). $\wedge$

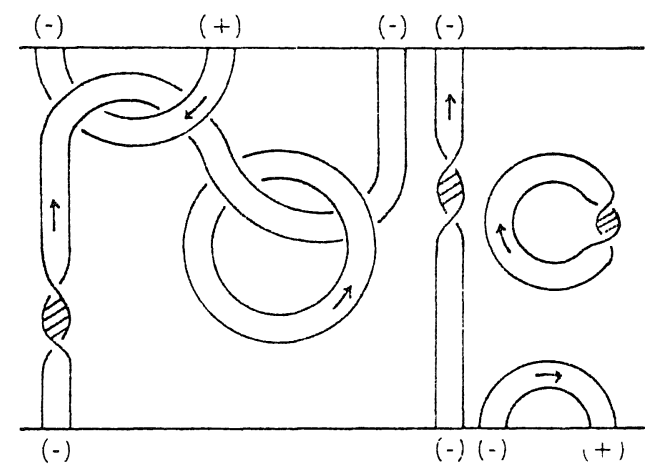

Fig. 1 
tangle is homogeneous if each twist of all ribbons and annuli in the tangle is a full twist. A ribbon tangle is directed if the cores of its ribbons and annuli are provided with directions. For each ribbon tangle we assign to each component a finite dimensional irreducible representation $V_{i}$ of $U_{t}$, where $i$ is called its colour. The procedure is called colouring and we denote it by $\lambda$. In Fig. 2, elementary coloured ribbon tangles are sketched. We consider ribbons which are called coupons. A small neighborhood of each coupon $Q$ is depicted in Fig. 3, where the rectangle illustrates the coupon. A colour of each coupon is a $C$-linear homomorphism defined from the colours and directions of the ribbons gluing to it. We add coupons to the tangle.

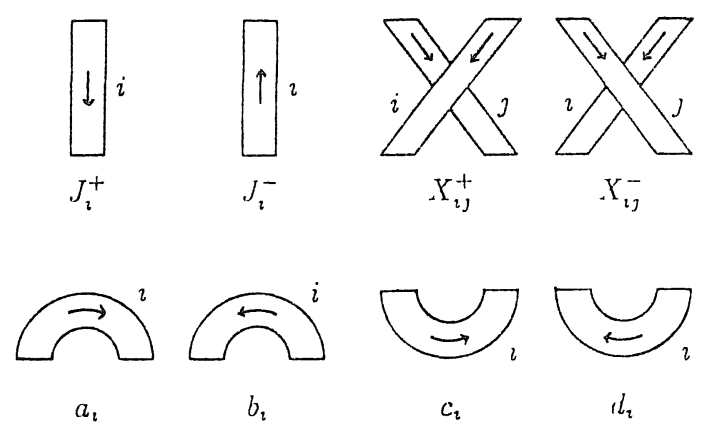

Fig. 2

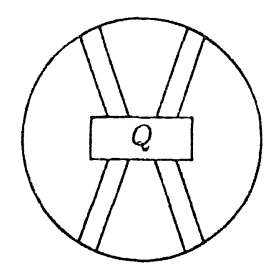

Fig. 3

Let us introduce the category $\mathscr{H}$ of ribbon graphs. The objects of $\mathscr{H}$ are sequences

$$
\eta=\left(\left(i_{1}, \varepsilon_{1}\right), \cdots,\left(i_{k}, \varepsilon_{k}\right)\right) \quad\left(i_{1}, \cdots, i_{k} \in I, \varepsilon_{1}, \cdots, \varepsilon_{k} \in\{1,-1\}\right) .
$$

We denote the set of such sequences by $N$. If $\eta, \eta^{\prime} \in N$, then a morphism $\eta \rightarrow$ $\eta^{\prime}$ is a coloured ribbon graph (considered up to isotopy) such that the sequence of colours and directions of the bottom (resp. top) ribbons is equal to $\eta$ (resp. $\left.\eta^{\prime}\right)$. The composition $\Gamma^{\prime} \circ \Gamma$ of such two morphisms $\Gamma: \eta \rightarrow \eta^{\prime}, \Gamma^{\prime}: \eta^{\prime} \rightarrow \eta^{\prime \prime}$ is the ribbon graph obtained by gluing the bottom ends of $\Gamma^{\prime}$ with the corresponding top ends of $\Gamma$. The tensor product of objects $\eta, \eta^{\prime}$ is their juxtaposition $\eta, \eta^{\prime}$ (see Fig. 4). 


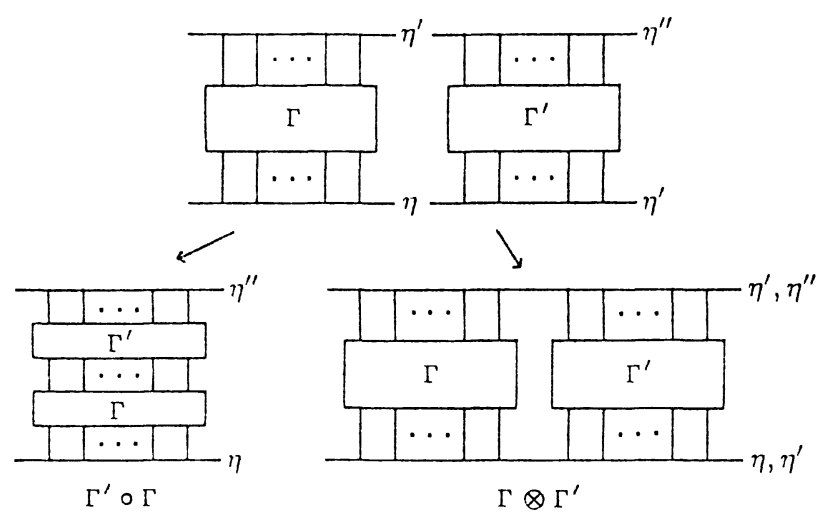

Fig. 4

\subsection{Invariants of Closed 3-Manifolds}

Reshetikhin and Turaev show that there exists a unique covariant functor from $\mathscr{H}$ to $\operatorname{Rep} U_{t}$ with five properties (see $\S 2.5$ in [13]). They define $U_{t}$-linear homomorphisms corresponding to elementary coloured ribbon graphs pictured in Fig. 2 and graphs pictured in Fig. 5 .

Since the graphs $J_{i}^{+}, J_{i}^{-}, X_{i j}^{+}, X_{i j}^{-}, a_{i}, b_{i}, c_{i}, d_{i}$ generated the category $\mathscr{A}$, the compositions and tensor products of the corresponding homomorphisms determine $F(\Gamma)$ for a coloured ribbon tangle $\Gamma$. In particular, a coloured $(0,0)$ ribbon tangle $\Gamma$ defines $C$-linear homomorphism $\boldsymbol{C} \rightarrow \boldsymbol{C}$, i.e. a multiplication by a certain element of $\boldsymbol{C}$. The element is a regular isotopy invariant of $\Gamma$. It is also denoted by $F(\Gamma)$.

Example 1.3. Let $\Gamma$ be a coloured $(0,0)$-ribbon tangle in Fig. 6. Then $F(\Gamma)=F\left(b_{i}\right) \circ F\left(c_{i}\right)$ and an easy computation shows $F(\Gamma)=\operatorname{dim}_{q} V_{i}$.

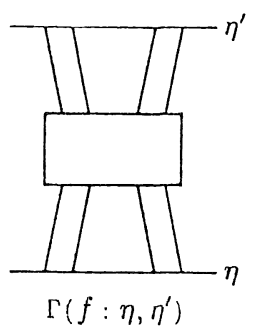

Fig. 5

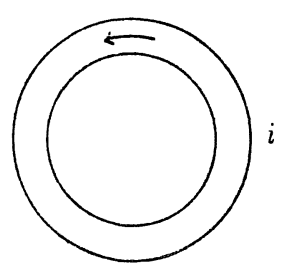

Fig. 6

Let us recall that $\operatorname{dim}_{q} V_{i}$ is equal to the quantum trace of identity homomorphism. The following lemma generalizes this computation.

Lemma 1.4. Let $\Gamma$ be a coloured $(k, k)$-ribbon graph which corresponds to an endomorphism of a certain sequence $\eta \in N$. Let $L$ be the coloured $(0,0)$-ribbon 


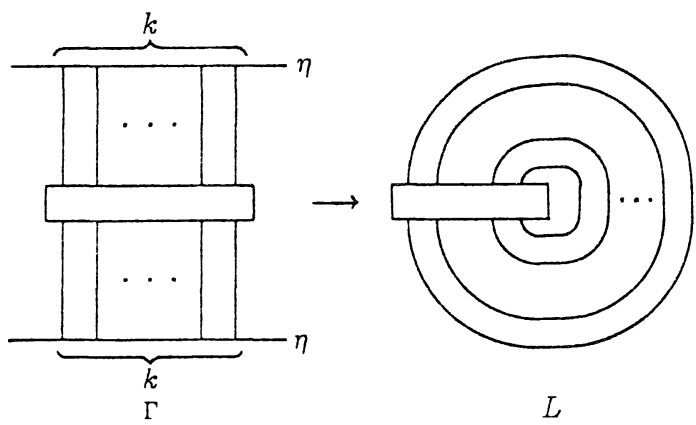

Fig. 7

tangle obtained by closing $\Gamma$ (see Fig. 7). Then $F(L)=\operatorname{tr}_{q} F(\Gamma)$.

We introduce the presentation of closed 3-manifolds via framed links. A framed link in the 3 -sphere is a finite collection $L$ of disjoint smoothly embedded circles $L_{1}, \cdots, L_{l}$ in $S^{3}$, each component $L_{k}$ of $L$ is provided with a framing which is an integer $n_{k}$. Let $\omega$ be an orientation of $L$. We may regard each component $L_{k}$ of the annulus with $n_{k}$ full twists. This identification gives us a $(0,0)$-ribbon tangle $\Gamma(L, \omega)$. The notation $\omega$ may be thought of as the directions of the annuli. Let $\lambda$ be a colouring of $\Gamma(L, \omega)$. Then $F(\Gamma(L, \omega, \lambda))$ is a regular isotopy invariant of coloured $(0,0)$-ribbon tangle $\Gamma(L, \omega, \lambda)$. By means of the above results, we define invariants of closed 3manifolds. The idea of their construction is reduced to the following theorem which relates framed links to closed 3-manifolds.

Theorem 1.5 (Lickorish [9]). Each closed connected oriented 3-manifold can be obtained by Dehn surgery on $S^{3}$ along a certain framed link.

Let $M$ be a closed connected oriented 3-manifold and $L$ a framed link in $S^{3}$ with components $L_{1}, \cdots, L_{l}$ and framing $n_{1}, \cdots, n_{l}$ which can be related to $M$ by the above theorem. Dehn surgery is the following process. We remove an open tubular neighborhood of each $L_{k}$ and on the resulting total boundary we glue $l$ solid tori such that their meridians are identified with the curves on the boundaries. We consider such a pair $(M, L)$. Let $\omega$ be an orientation of the framed link $L$. By $\operatorname{col}(L)$ we denote the set of colourings of the $(0,0)$ ribbon tangle $\Gamma(L, \omega)$. Put

$$
F(M, L)=C^{\sigma(L)} \sum_{\lambda \in \operatorname{col}(L)} \prod_{k=1}^{l} d_{\lambda\left(L_{k}\right)} F(\Gamma(L, \omega, \lambda)) \in C .
$$

Here $C, d_{k}(k=0, \cdots, r-2)$ are constants contained in the data of the modular Hopf algebra $U_{t}$ and given by the following formulas:

$$
C=\exp (-\sqrt{-1} d),
$$




$$
d_{k}=\sqrt{\frac{2}{r} \sin \frac{m(k+1) \pi}{r},}
$$

where

$$
d=\varphi-\frac{3 \pi m}{2 r}+\frac{\pi}{2}
$$

the number $\varphi$ being determined from the following Gauss sum

$$
\sqrt{2 r} \exp (\sqrt{-1} \varphi)=\sum_{k=0}^{2 r-1} \exp \left(\sqrt{-1} \pi k^{2} m / 2 r\right) .
$$

The notation $\sigma(L)$ stands for the signature of the linking matrix of the framed link $L$. We remark that the normalization coincides with that in [8].

Theorem 1.6 (Reshetikhin-Turaev). For a closed connected oriented 3manifold $M, F(M, L)$ is a topological invariant of $M$.

We may denote $F(M, L)$ by $F(M)$. The invariant is multiplicative with respect to a connected sum :

$$
F\left(M_{1} \# M_{2}\right)=F\left(M_{1}\right) F\left(M_{2}\right) .
$$

We have the following relations between invariants with opposite orientations

$$
F(M)=\overline{F(-M)},
$$

where the bar is the complex conjugation.

Example 1.7. The formula (1.3.6) implies that $F\left(S^{3}\right)=1$.

Since $S^{2} \times S^{1}$ is obtained by Dehn surgery on $S^{3}$ along an unknotted circle with framing 0 , we have

$$
\begin{aligned}
F\left(S^{2} \times S^{1}\right) & =\sum_{i=0}^{r-2} d_{i} \operatorname{dim}_{q} V_{i} \\
& =\sqrt{\frac{r}{2}}\left(\sin \frac{m \pi}{r}\right)^{-1} .
\end{aligned}
$$

Here we used the equation $\operatorname{dim}_{q} V_{i}=\sin (m(i+1) \pi / r) / \sin (m \pi / r)$. In the case $m=$ $1, F\left(S^{2} \times S^{1}\right)$ is equal to Kohno's invariant $\phi_{K}\left(S^{2} \times S^{1}\right)$ with $K=r+2$.

Let $M$ be a closed connected oriented 3-manifold and $T$ be a coloured $(0,0)$ ribbon tangle in $M$. As above, let us present $M$ as the result of surgery on $S^{3}$ along a framed link $L$ with components $L_{1}, \cdots, L_{l}$. The ribbon tangle $T \cup \Gamma(L, \omega, \lambda)$ may be thought of as a coloured $(0,0)$-ribbon tangle in $S^{3}$. We put

$$
F(M, T)=C^{\sigma(L)} \sum_{\lambda \in \operatorname{col}(L)} \prod_{k=1}^{l} d_{\lambda\left(L_{k}\right)} F(T \cup \Gamma(L, \omega, \lambda)) .
$$

In particular, we have $F\left(S^{3}, T\right)=F(T)$. 


\section{§2. A Representation of $S L(2, Z)$}

Using the invariants defined in $\S 1$, we establish a projectively linear representation of $S L(2, Z)$. Let $M_{1}$ be the mapping class group of torus $T^{2}$. We fix a basis $a, b$ in $H_{1}\left(T^{2}\right) \cong Z \oplus Z$ as depicted in Fig. 8 .

The group $M_{1}$ may be canonically identified with $S L(2, \mathbb{Z})$. A presentation of $S L(2, Z)$ is given by

$$
S L(2, Z)=\left\langle S, T ; S^{4}=I,(S T)^{3}=S^{2}\right\rangle,
$$

where $S=\left(\begin{array}{cc}0 & -1 \\ 1 & 0\end{array}\right), T=\left(\begin{array}{ll}1 & 1 \\ 0 & 1\end{array}\right)$. Let $Z\left(T^{2}\right)$ be an $(r-1)$-dimensional vector space over $C$ and $\left\{e_{0}, e_{1}, \cdots, e_{r-2}\right\}$ a basis of the vector space. We associate to each $e_{i}$ a solid torus $U_{i}$ with an annulus $T_{i}$ in the interior, depicted in Fig. 9. We suppose that the colour of annulus $T_{i}$ is $i \in\{0, \cdots, r-2\}$ and the direction as in Fig. 9. We construct a projectively linear representation

$$
\rho: S L(2, \boldsymbol{Z}) \longrightarrow G L\left(Z\left(T^{2}\right)\right) /\langle C\rangle,
$$

where $C$ is given by (1.3.2) and $\langle C\rangle$ means the cyclic group generated by $C \cdot I$, when $I$ denotes the identity matrix.

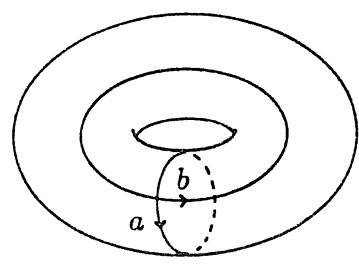

Fig. 8

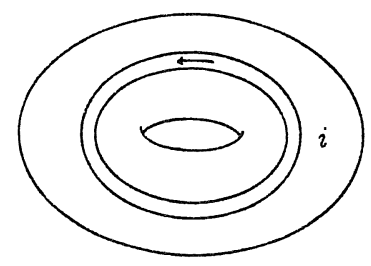

Fig. 9

For any element $X$ of $S L(2, Z)$, put

$$
\rho(X) e_{j}=\sum_{i=0}^{r-2} X_{i j} e_{i} .
$$

Let $[h]$ be an isotopy class in $M_{1}$ corresponding to $X$. The map $h$ is a degree 1 homeomorphism $T^{2} \rightarrow T^{2}$. We identify $\partial U_{i}$ and $\partial U_{j}$ using $h$. The resulting closed connected 3-manifold with the $(0,0)$-ribbon tangle consisting of two annuli $T_{i}, T_{j}$ is denoted by $M_{X}$. Then $X_{i j}$ in (2.2) is defined by the following formula:

$$
X_{i \jmath}=F\left(M_{X}, T_{i} \cup T_{\jmath}\right) / F\left(S^{2} \times S^{1}\right) .
$$

Clearly, it follows from the definition that $X_{\imath j}$ does not depend on the choice of the representative element of the isotopy class.

Theorem 2.1. The following homomorphism constructed above is a projectively linear representation.

$$
\rho: S L(2, Z) \longrightarrow G L\left(Z\left(T^{2}\right)\right) /\langle C\rangle,
$$


where $\langle C\rangle$ means the cyclic group generated by $C \cdot I$ in $G L\left(Z\left(T^{2}\right)\right)$ with $C$ given by (1.3.2). The values of $S_{i j}, I_{i j}$ and $T_{i j}$ are given by the following formulas:

$$
\begin{aligned}
& S_{i \jmath}=\sqrt{\frac{2}{r}} \sin \frac{m(i+1)(j+1) \pi}{r}, \\
& I_{i \jmath}=\delta_{i \jmath}, \\
& T_{i j}=t^{i(\imath+2)} \delta_{i \jmath} .
\end{aligned}
$$

Proof. Let $C_{h}$ be the mapping cylinder of the homeomorphism $h: T^{2} \rightarrow T^{2}$. We parametrize $T^{2} \times\{0\}$ via the identity and $T^{2} \times\{1\}$ via $h$. Using the parametrization, we glue solid tori $U_{i}$ and $U_{j}$ to $C_{h}$. Then we obtain a closed 3 manifold $M_{X}$ with two annuli $T_{i}, T_{j}$. If isotopy classes $[h],[g] \in M_{1}$, then the cylinder $C_{h^{\circ} g}$ splits into a composition of $C_{g}$ and $C_{h}$. Let $X$ (resp. $Y$ ) be an element $S L(2, C)$ corresponding to the isotopy class [h] (resp. [g]). Gluing $U_{\imath}$ and $U_{\jmath}$ to the composition $C_{h} C_{g}$, we obtain a closed 3-manifold $M_{X Y}$. Let $L_{X}$ (resp. $L_{Y}$ ) be a framed link in $S^{3}$ which we obtain $M_{X}$ (resp. $M_{Y}$ ) by Dehn surgery along. We consider $\left(M_{X}, T_{\imath} \cup T_{k}\right),\left(M_{Y}, T_{k} \cup T_{\jmath}\right)$ and $\left(M_{X Y}, T_{i} \cup T_{\jmath}\right)$. Connecting the annulus $T_{k}$ in $L_{X} \cup T_{k} \cup T_{2}$ in $S^{3}$ with the annulus $T_{k}$ in $L_{Y} \cup T_{k} \cup T_{\jmath}$, a new ribbon tangle $L_{X} \cup L_{Y} \cup L_{0} \cup T_{\imath} \cup T_{j}$ is constructed, where $L_{0}$ is a circle determined by $T_{k}$. We denote it by $L$. We can get $\left(M_{X Y}, T_{i} \cup T_{\jmath}\right)$ by Dehn surgery along the framed link $L$ in $S^{3}$. From the definition of $d_{k}$ and a homomorphism corresponding to the composition of tangles, it follows that

$$
F\left(M_{X Y}, T_{\imath} \cup T_{\jmath}\right)=C^{n} \sum_{k=0}^{r-2} F\left(M_{X}, T_{i} \cup T_{k}\right) F\left(M_{Y}, T_{k} \cup T_{\jmath}\right)
$$

with $n=\sigma(L)-\sigma\left(L_{X}\right)-\sigma\left(L_{Y}\right)$. This shows that $\rho\left(X Y^{\gamma}\right)=C^{n} \rho(X) \rho(Y)$.

Let us compute $S_{\imath \jmath}, I_{\imath \jmath}$, and $T_{\imath \jmath}$.

(1) the case $X=S$

$M_{S}$ is the 3-sphere $S^{3}$. 'Two annuli $T_{\imath}, T$, are linked in $M_{S}$ and make up the Hopf link (see Fig. 10).

Therefore we get $F\left(M_{S}, T_{\imath} \cup T_{\jmath}\right)=F\left(T_{\imath} \cup T_{\jmath}\right)$. One computes

$$
F\left(T_{\imath} \cup T_{\jmath}\right)=\sin \frac{m(i+1)(j+1) \pi}{r} / \sin \frac{m \pi}{r} .
$$

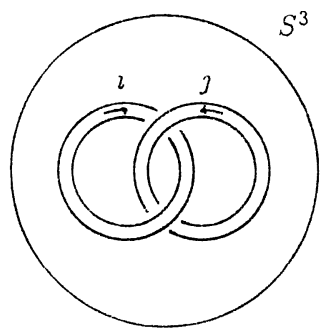

Fig. 10

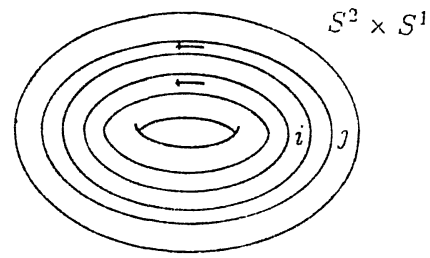

Fig. 11 
Applying (2.3) with (1.3.7) and (2.4), we get

$$
S_{i j}=\sqrt{\frac{2}{r}} \sin \frac{m(i+1)(j+1) \pi}{r} .
$$

(2) the case $X=I$

$M_{I}$ is $S^{2} \times S^{1}$. In $M_{I}, T_{i}$ and $T_{\partial}$ are unlinked unknotted annuli with no twists (see Fig. 11). Let us consider $S^{3}$ with the above annuli and the unknotted circle $L$ that links a pair of the annuli and that has the zero framing as illustrated in Fig. 12a.

The Dehn surgery on $S^{3}$ along $L$ produces $S^{2} \times S^{1}$ with $T_{\imath}$ and $T_{\text {, depicted }}$ in Fig. 11. To calculate $F\left(T_{\imath} \cup T, \cup \Gamma(L, \omega, \lambda)\right)$, we can use the formula (1.1.2)

$$
V_{\imath} \otimes V_{j}=\left(\oplus_{k} V_{k}\right) \oplus Z_{i \jmath} .
$$

Let us replace $T_{\imath}$ and $T_{j}$ with a unknotted annulus $T_{k}$ which runs parallel to $T_{\imath}$ and $T_{j}$ (Fig. 12b). We assume that $T_{k}$ has a colour $k$ and the same direction as two annuli. Then $T_{k} \cup \Gamma(L, \omega, \lambda)$ is a $(0,0)$-ribbon tangle in $S^{3}$.

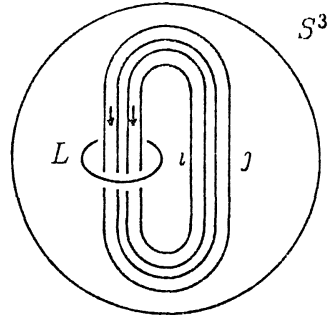

Fig. 12a

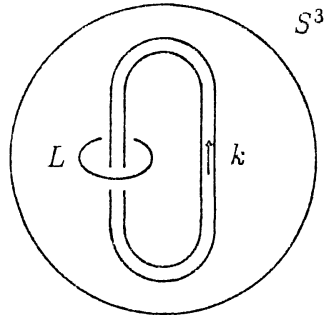

Fig. 12b

The property (1.1.15) of the $U_{t}$-module $Z_{\imath \jmath}$ ensures the equation

$$
F\left(T_{i} \cup T_{j} \cup \Gamma(L, \omega, \lambda)\right)=\sum_{k} F\left(T_{k} \cup \Gamma(L, \omega, \lambda)\right),
$$

where the summation runs over $k$ satisfying (1.1.13) and (1.1.14). As $T_{k} \cup$ $\Gamma(L, \omega, \lambda)$ is the Hopf link, we can apply (2.4) to the computation of $F\left(T_{k} \cup\right.$ $\Gamma(L, \omega, \lambda))$. If $\lambda(L)=l$, then we obtain

$$
F\left(T_{k} \cup \Gamma(L, \omega, \lambda)\right)=F\left(S^{2} \times S^{1}\right) \sqrt{\frac{2}{r}} \sin \frac{m(k+1)(l+1) \pi}{r} .
$$

Thus, we get

$$
I_{i j}=\frac{1}{F\left(S^{2} \times S^{1}\right)} \sum_{l=0}^{r-2} d_{l}\left(\sum_{k} F\left(S^{2} \times S^{1}\right) \sqrt{\frac{2}{r}} \sin \frac{m(k+1)(l+1) \pi}{r}\right),
$$

where $k$ satisfies the conditions (1.1.13) and (1.1.14). We have the following formula :

$$
\sum_{l=0}^{r-2} \sin \frac{m(i+1)(l+1) \pi}{r} \sin \frac{m(l+1)(j+1) \pi}{r}=\frac{r}{2} \delta_{\imath \jmath} .
$$


Using (2.8), we show the formula:

$$
I_{i \jmath}=\frac{2}{r} \sum_{k} \frac{r}{2} \delta_{0 k}
$$

The condition (1.1.13) of $k$ asserts that $k$ is equal to zero if and only if $i=j$. Therefore we get

$$
I_{i \jmath}=\delta_{\imath \jmath}
$$

(3) the case $X=T$

$M_{T}$ is also $S^{2} \times S^{1}$. But the unknotted annulus $T_{i}$ with no twists links the unknotted annulus $T_{j}$ with one full twist (Fig. 13). To obtain $\left(M_{T}, T_{i} \cup T_{\jmath}\right)$, we start from $S^{3}$ with the two above annuli $T_{i}$ and $T$, and with an unknotted circle $L$ which has the zero framing and which links them (Fig. 14a). Carrying out the Dehn surgery on $S^{3}$ along the circle $L$ turns $S^{3}$ into $M_{T} \cong S^{2} \times S^{1}$.

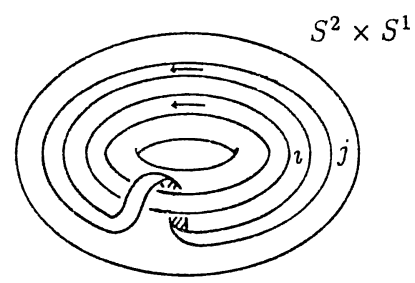

Fig. 13

One claims that we can use of the idea of the case $X=I$ to calculate $F\left(T_{\imath} \cup T, \cup \Gamma(L, \omega, \lambda)\right)$. We deform the annulus $T_{i}$ adding the same twist as the annulus $T_{\jmath}$. One denotes the resulting annulus by $T_{i}^{\prime}$. The computaion in $[13$, the proof of Lemma 7.1] implies

$$
F\left(T_{i}^{\prime} \cup T_{j} \cup \Gamma(L, \omega, \lambda)\right)=\left(v_{\imath}\right)^{-1} F\left(T_{i} \cup T, \cup \Gamma(L, \omega, \lambda)\right),
$$

where $v_{\imath}=t^{2(i+2)}$. A full twist can be expressed by a curl (Fig. 14b). It follows from it that we can turn $T_{\imath}^{\prime} \cup T$, into two parallel annuli with no twists (Fig. 14c).

Let $T_{k}$ be an annulus of colour $k$ provided with the same twist and direction as two annuli. We replace two annuli by $T_{k}$ (Fig. 14d).

Then, applying Theorem 1.2, one gets the following equation

$$
F\left(T_{i}^{\prime} \cup T, \cup \Gamma(L, \omega, \lambda)\right)=\sum_{\substack{k \\ i=j \leq k \leq i+\jmath \\ i+j+k \in 2 \\ i+j+k \leq 2(r-2)}} F\left(T_{k} \cup \Gamma(L, \omega, \lambda)\right),
$$

Thus

$$
T_{i J}=\frac{1}{F\left(S^{2} \times S^{1}\right)} \sum_{l=0}^{r-2} d_{l} v_{i} \sum_{k} F\left(T_{k} \cup \Gamma(L, \omega, \lambda)\right)
$$

here $\lambda(L)=l$. Substituting $v_{\imath}=t^{i(i+2)}$, we obtain

$$
T_{i j}=t^{i(i+2)} \delta_{i j} .
$$




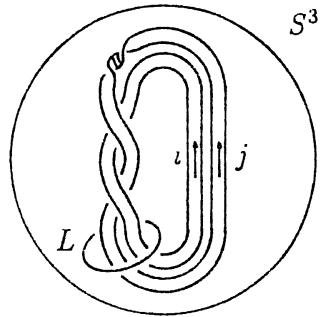

Fig. 14a

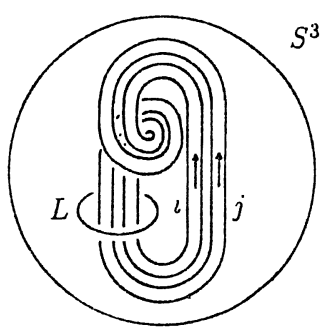

Fig. 14c

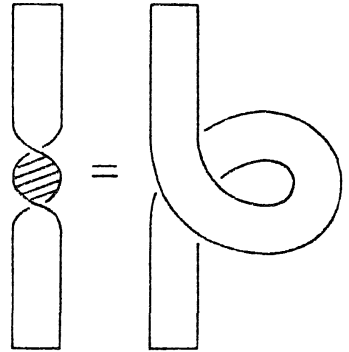

Fig. 14b

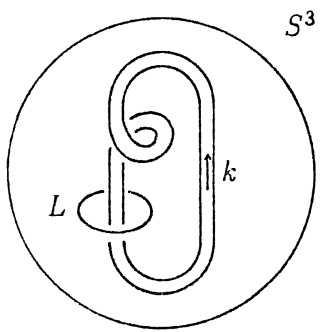

Fig. 14d

We put $I_{i d}=\left(I_{i j}\right), S=\left(S_{i j}\right)$ and $T=\left(T_{i \jmath}\right)$. They are $(r-1) \times(r-1)$ matrices.

We show the following:

$$
S^{4}=I_{i d} \quad \bmod C \cdot I
$$

$$
(S T)^{3}=S^{2} \quad \bmod C \cdot I \text {. }
$$

One easily computes

$$
S^{2}=I_{i d}
$$

Note that the equation $(S T)^{3}=S^{2}$ is equivalent to the equation $S T S=T^{-1} S T^{-1}$. It is easy to compute that an $(i, j)$-entry of $T^{-1} S T^{-1}$ is

$$
\sqrt{\frac{2}{r}} t^{-i(\imath+2)-\jmath(j+2)} \sin \frac{m(i+1)(j+1) \pi}{r} .
$$

Using $t=\exp (\pi \sqrt{-1} m / 2 r)$ and Gauss sum (1.3.5), an $(i, j)$-entry of STS is

$$
C \sqrt{\frac{2}{r}} t^{-i(i+2)-j(j+2)} \sin \frac{m(i+1)(j+1) \pi}{r} .
$$

It follows from (2.14) and (2.15) that

$$
S T S=T^{-1} S T^{-1} \cdot C I_{i d} .
$$

(2.13) implies (2.11) and (2.16) implies (2.12). 


\section{§3. Proof of Verlinde's Formula}

As another application of the invariants given in $\S 1$, we prove 'Verlinde's formula' (see [15]). It is given by the following formula.

$$
\frac{S_{i \jmath} S_{i k}}{S_{i 0}}=\sum_{l=0}^{r-2} S_{i l} N_{l \jmath k}
$$

where $m$ and $r$ are mutually prime integers with odd $m, 1 \leqq m \leqq 2 r-1,2 \leqq r$, and

$$
\begin{aligned}
& S_{i \jmath}=\sqrt{\frac{2}{r} \sin \frac{m(i+1)(j+1) \pi}{r},} \\
& N_{i \jmath k}= \begin{cases}1 & \text { if }|i-j| \leqq k \leqq i+j, i+j+k \in 2 \boldsymbol{Z}, i+j+k \leqq 2(r-2) \\
0 & \text { otherwise. }\end{cases}
\end{aligned}
$$

Proof of Verlinde's formula. Let us consider $S^{2} \times S^{1}$ with three parallel non-twisted annuli $T_{l}, T_{\jmath}, T_{k}$ in the interior (see Fig. 15). The directions of them is as in Fig. 15 and the colour of $T_{l}$ (resp. $T_{\jmath}, T_{k}$ ) is $l$ (resp. $j, k$ ).

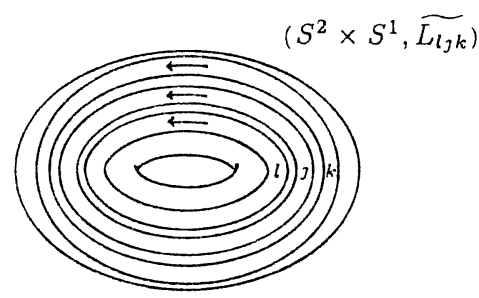

Fig. 15

We call this configuration of three annuli $\widetilde{L_{l \jmath k}}$. The idea of the proof is to evaluate $F\left(S^{2} \times S^{1}, \widetilde{L_{l \jmath k}}\right)$ in two ways.

Let us begin with the surgery representation of $\left(S^{2} \times S^{1}, \widetilde{L_{l \jmath k}}\right)$. Let $L$ be an unknotted circle with the zero framing which links $\widetilde{L_{l j k}}$ in $S^{3}$ (Fig. 16a). The Dehn surgery on $S^{3}$ along the circle $L$ produces $\left(S^{2} \times S^{1}, \widetilde{L_{l j k}}\right)$.

In the first evaluation, we use an analogue of the computation of $I_{i j}$ and $T_{i}$, in $\S 2$. We replace $T_{j}$ and $T_{k}$ by an unknotted non-twisted annulus $T_{p}$ with colour $p$ and the same direction as them (Fig. 16b). Then applying Theorem 1.2 with $i$ replaced by $l$, we obtain the following equation:

$$
F\left(\widetilde{L_{l \jmath k}} \cup \Gamma(L, \omega, \lambda)\right)=\sum_{p} F\left(T_{l} \cup T_{p} \cup \Gamma(L, \omega, \lambda)\right) .
$$

Here $p$ satisfies the conditions (1.1.13) and (1.1.14) replaced $i$ by $p$. 


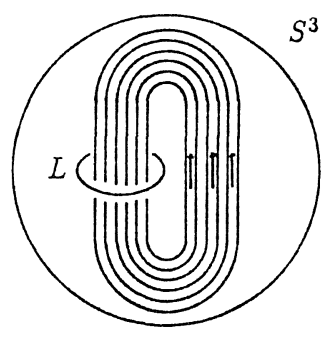

Fig. 16a

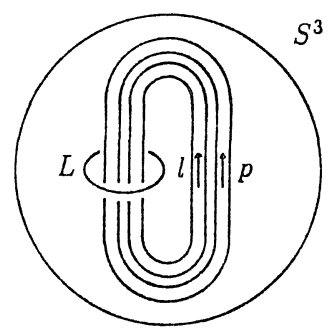

Fig. 16b

Then we can apply the formula (2.9) to the computation. Thus we get

$$
\begin{aligned}
F\left(S^{2} \times S^{1}, \widetilde{L_{l \jmath k}}\right. & =\sum_{t=0}^{r-2} d_{t}\left(\sum_{p} F\left(T_{l} \cup T_{p} \cup \Gamma(L, \omega, \lambda)\right)\right) \\
& =F\left(S^{2} \times S^{1}\right) \sum_{\substack{p \\
1 i-j \leq p \leq j+k \\
p+j+p \in 2 Z \\
p+j+k \leqq 2}} \delta_{l, p} .
\end{aligned}
$$

It follows from the condition of $p$ that

$$
F\left(S^{2} \times S^{1}, \widetilde{L_{l \jmath k}}\right)=F\left(S^{2} \times S^{1}\right) N_{l \jmath k} .
$$

To evaluate $F\left(S^{2} \times S^{1}, \widetilde{L_{l j k}}\right)$ in the second way, we rotate the $(0,0)$-ribbon tangle $\widetilde{L_{l j k}} \cup \Gamma(L)$ in $S^{3}$ (Fig. 17a). The result may be thought of as the closure of the $(1,1)$-ribbon tangle $B_{l j k}^{t}$ illustrated in Fig. $17 \mathrm{~b} . \quad F\left(B_{l j k}^{t}\right)$ is the homomorphism $V_{t} \rightarrow V_{t}$. Moreover, it may be thought of as the composition of three homomorphisms determined by $(1,1)$-ribbon tangles $\tau_{l}^{t}, \tau_{\jmath}^{t}, \tau_{k}^{t}$ illustrated in Fig. 17c.

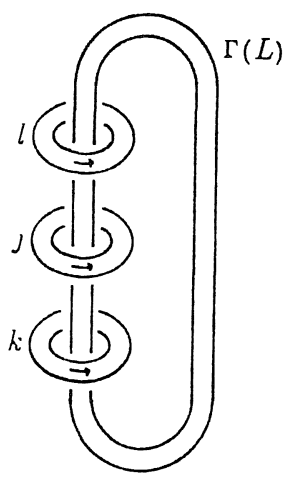

Fig. 17a

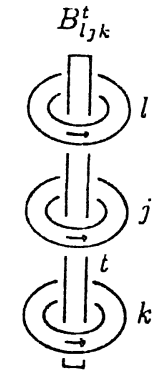

Fig. 17b
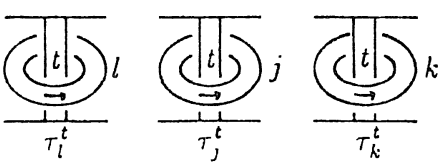

Fig. 17c

The map $F\left(\tau_{l}^{t}\right)$ is a $C$-linear homomorphism $V_{t} \rightarrow V_{t}$ and $V_{t}$ is irreducible, by Schur's lemma, it is a multiplication by an element of $C$. We denote this element by $b_{l}^{t}$. Similarly, $F\left(\tau_{j}^{t}\right)$ (resp. $F\left(\tau_{k}^{t}\right)$ ) is a multiplication by an element 
$b_{j}^{t}$ (resp. $b_{k}^{t}$ ) of $C$. The closure of the $(1,1)$-ribbon tangle $\tau_{l}^{t}$ makes up the Hopf link. We denote this invariant by $s_{t l}$. Analogously, the invariant which corresponds to $\tau_{j}^{t}$ (resp. $\tau_{k}^{t}$ ) is denoted by $s_{t \jmath}$ (resp. $s_{t k}$ ). Using (2.4), we derive

$$
s_{t \mu}=\sin \frac{m(t+1)(\mu+1) \pi}{r} / \sin \frac{m \pi}{r},
$$

where $\mu \in\{l, j, k\}$. Note that $s_{t 0}=\operatorname{dim}_{q} V_{t}$. Then Lemma 1.5 shows that

$$
s_{t / \ell}=b_{\mu}^{t} \operatorname{dim}_{q} V_{t}=b_{\mu}^{t} s_{t 0} .
$$

The above discussion and (3.6) imply that

$$
\begin{aligned}
F\left(B_{l \jmath k}^{t}\right) & =\operatorname{tr}_{q}\left(F\left(\tau_{l}^{t}\right) \circ F\left(\tau_{\jmath}^{t}\right) \circ F\left(\tau_{k}^{t}\right)\right) \\
& =b_{l}^{t} b_{\jmath}^{t} b_{k}^{t} \operatorname{dim}_{q} V_{t} .
\end{aligned}
$$

Using (3.4) and (3.5),

$$
\begin{aligned}
F\left(S^{2} \times S^{1}, \widetilde{L_{l \jmath k}}\right) & =\sum_{t=0}^{r-2} d_{t} F\left(B_{l \jmath k}^{t}\right) \operatorname{dim}_{q} V_{t} \\
& =\sum_{t=0}^{r-2} d_{t} \frac{s_{t l} s_{t,} s_{t k}}{\left(s_{t 0}\right)^{2}} .
\end{aligned}
$$

Multiplying (3.3) and (3.6) by $s_{\imath \iota}$ and summing up over $l=0, \cdots, r-2$, we get

$$
\sum_{l=0}^{r-2} s_{\imath l} F\left(S^{2} \times S^{1}\right) N_{l \jmath k}=d_{i}\left(\sin \frac{m \pi}{r}\right)^{-2} \frac{r}{2} \frac{s_{i \jmath} s_{i k}}{\left(s_{i 0}\right)^{2}} .
$$

We remark that

$$
\begin{aligned}
d_{i} & =\sqrt{\frac{2}{r}} \sin \frac{m(i+1) \pi}{r} \\
& =\sqrt{\frac{2}{r}} s_{\imath 0} \sin \frac{m \pi}{r} .
\end{aligned}
$$

Substituting (3.8) in (3.7), we obtain

$$
\sum_{l=0}^{r-2} s_{i l} F\left(S^{2} \times S^{1}\right) N_{l \jmath k}=F\left(S^{2} \times S^{1}\right) \frac{s_{\imath \jmath} s_{\imath k}}{s_{i 0}} .
$$

The value $S_{i}$, is related to $s_{i}$, by the formula

$$
s_{i j}=\sqrt{\frac{r}{2}}\left(\sin \frac{m \pi}{r}\right)^{-1} S_{i j} .
$$

Thus (3.9) implies (3.1).

\section{\$4. Ising model}

Instead of the modular Hopf algebra $U_{t}$ in $\S 1$, we consider a fusion algebra $A$ over $C$ corresponding to 'Ising model' [10]. It has generators $1, \sigma, \phi$ and their relations are 


$$
\psi \cdot \psi=1, \quad \psi \cdot \sigma=\sigma \cdot \psi=\sigma, \quad \sigma \cdot \sigma=1+\psi .
$$

It is known that this algebra describes the fusion rule for the critical Ising model (see for example [2]). The algebra $A$ has the conformal dimensions:

$$
\Delta_{1}=0, \quad \Delta_{\sigma}=\frac{1}{16}, \quad \Delta_{\psi}=\frac{1}{2} .
$$

It is analogous to the case $m=1$ and $r=4$ in the algebra $U_{t}$. But the element in $U_{t}$ corresponding to $\Delta_{\sigma}$ has a different value. Using the algebra $A$, we construct invariants of links and 3-manifolds. As an application, we obtain a projectively linear representation of $S L(2, \boldsymbol{Z})$ and an equation similar to Verlinde's formula.

Let $L$ be a framed link in $S^{3}$ with $m$ components $L_{1}, \cdots, L_{m}$ and $\Gamma(L)$ a diagram of $L$. We assign to each component $L_{\imath}$ of $L$ one of generators of $A$. We denote the assignment by $\lambda$, which gives a colouring

$$
\left\{L_{1}, \cdots, L_{m}\right\} \longrightarrow\{1, \sigma, \phi\} .
$$

Next, we assign an element (or colour) of the set $\{1, \sigma, \phi\}$ to each region of $\Gamma(L)$. This assignment follows the fusion rule (4.1) in the following sense. We assign 1 to the unbounded region. Let $A_{1}$ and $A_{2}$ be adjacent regions and the component of $L$ between $A_{1}$ and $A_{2}$ have colour $j \leqq\{1, \sigma, \phi\}$. A colour $a_{\imath}$ of the region $A_{i}$ for $i=1,2$ satisfies the following equation:

$$
a_{2}= \begin{cases}1 \text { or } \phi & \text { if } a_{1}=j=\sigma, \\ a_{1} \cdot j & \text { otherwise. }\end{cases}
$$

When a colour of the link $L$ in Fig. 18 is $\sigma$, all the colourings of regions are given in Fig. 19.

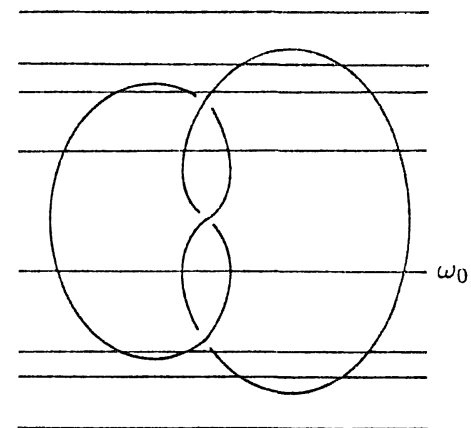

Fig. 18

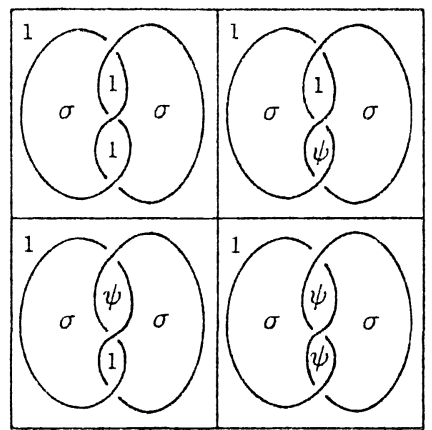

Fig. 19

Any horizontal line $\omega$ which avoids crossings and extreme (maximum or minimum) points hits $\Gamma(L)$ in a finite number of points as pictured in Fig. 18. 
We assume that the critical points occur in distinct levels. We may then decompose the diagram $\Gamma(L)$ level by level as the composite of a number of elementary diagrams, which each diagram contains just one critical point. In each elementary diagram, one of the four diagrams (b)-(d) shown in Fig. 20 exists, while the rest of the diagrams consists of the strings passing from the top to the bottom without crossings as (a) shown in Fig. 20.

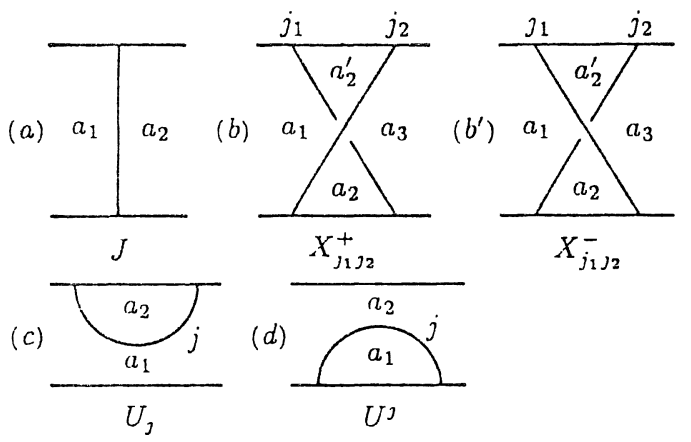

Fig. 20

We associate to any horizontal line $\omega$ a vector space $V_{\omega}$. If the colours of the regions which the line $\omega$ passes through are in the order $1, a_{1}, \cdots, a_{l}, 1$, the vector space $V_{\omega}$ has a base element $e_{1} \otimes e_{a_{1}} \otimes \cdots \otimes e_{a_{l}} \otimes e_{1}$. If the colour of the link $L$ in Fig. 18 is $\sigma$, the vector space $V_{\omega_{0}}$ corresponding to the line $\omega_{0}$ has a basis

$$
\left\{e_{1} \otimes e_{\sigma} \otimes e_{1} \otimes e_{\sigma} \otimes e_{1}, e_{1} \otimes e_{\sigma}\left(\otimes e_{\mu}, \otimes e_{\sigma} \otimes e_{1}\right\}\right.
$$

Finally, we associate a $\boldsymbol{C}$-linear homomorphism $\boldsymbol{C} \rightarrow \boldsymbol{C}$ to the diagram $\Gamma(L)$. Let us denote a linear operator over $\boldsymbol{C}$ for a diagram $T$ by $F_{T}$. The operators for elementary diagrams shown in Fig. 20 are determined by the following formulas. The notation $a_{\imath}$ (resp. $j_{\imath}$ ) represents a colour of a region (resp. a string) in Fig. 20.

(a) $F_{J}$ is the identity homomorphism $F_{J}: e_{a_{1}} \otimes e_{a_{2}} \mapsto e_{a_{1}} \otimes e_{a_{2}}$.

(b) Let us consider a tangle diagram pictured in Fig. 21.

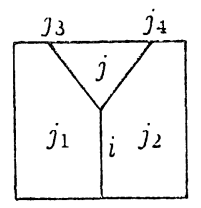

Fig. 21 
The operator for this diagram is a multiplication by $F_{i j}\left[\begin{array}{ll}j_{2} & j_{3} \\ j_{1} & j_{4}\end{array}\right]$. The matrix $F\left[\begin{array}{ll}j_{2} & j_{3} \\ j_{1} & j_{4}\end{array}\right]=\left(F_{i j}\left[\begin{array}{ll}j_{2} & j_{3} \\ j_{1} & j_{4}\end{array}\right]\right)_{i j}$ is called 'fusing matrix'. Explicitly, we have

$$
F\left[\begin{array}{ll}
\sigma & \sigma \\
\sigma & \sigma
\end{array}\right]=\frac{1}{\sqrt{2}}\left(\begin{array}{rr}
1 & 1 \\
1 & -1
\end{array}\right), \quad F\left[\begin{array}{ll}
\sigma & \phi \\
\psi & \sigma
\end{array}\right]=F\left[\begin{array}{ll}
\psi & \sigma \\
\sigma & \psi
\end{array}\right]=-1
$$

and the other fusing matrices $F\left[\begin{array}{ll}j_{2} & j_{3} \\ j_{1} & j_{4}\end{array}\right]=1$. The operator for (b) in Fig. 20 is determined from the fusing matrix and the conformal dimensions $\Delta_{1}, \Delta_{\sigma}, \Delta_{\psi}$, and we denote the entry of the matrix for the operator by $B_{i j}\left[\begin{array}{ll}j_{2} & j_{3} \\ j_{1} & j_{4}\end{array}\right]$. The matrix $B\left[\begin{array}{ll}j_{2} & j_{3} \\ j_{1} & j_{4}\end{array}\right]=\left(B_{i j}\left[\begin{array}{ll}j_{2} & j_{3} \\ j_{1} & j_{4}\end{array}\right]\right)_{i j}$ is called 'braiding matrix' and related to the fusing matrix and $\Delta_{1}, \Delta_{\sigma}, \Delta_{\psi}$ by the following equation:

$$
B\left[\begin{array}{cc}
j_{2} & j_{3} \\
j_{1} & j_{4}
\end{array}\right]=F^{-1}\left[\begin{array}{cc}
j_{2} & j_{3} \\
j_{1} & j_{4}
\end{array}\right] \varepsilon \operatorname{diag}_{k}\left(\exp \pi \sqrt{-1}\left(\Delta_{k}-\Delta_{\jmath_{2}}-\Delta_{\jmath_{3}}\right)\right) F\left[\begin{array}{ll}
j_{2} & j_{3} \\
j_{1} & j_{4}
\end{array}\right],
$$

where

$$
k= \begin{cases}1 \text { or } \phi & \text { if } j_{2}=j_{3}=\sigma, \\ j_{2} \cdot j_{3} & \text { otherwise }\end{cases}
$$

and

$$
\varepsilon= \begin{cases}-1 & \text { if }\left\{j_{2}, j_{3}\right\}=\{\sigma, \phi\}, \phi \in\left\{j_{1}, j_{4}\right\}, \\ 1 & \text { otherwise. }\end{cases}
$$

These fusing matrices and braiding matrices satisfy the pentagon relation and the hexagon relation (see [10]).

(1) the case $j_{1} \neq \sigma$ or $j_{2} \neq \sigma$

$F_{X_{j_{1} j_{2}}^{+}}$is a multiplication by scalar $B\left[\begin{array}{ll}j_{2} & j_{2} \\ a_{1} & a_{3}\end{array}\right]=\varepsilon \exp \pi \sqrt{-1}\left(\Delta_{k}-\Delta_{\jmath_{1}}-\Delta_{1_{2}}\right)$, where $k=j_{1} \cdot j_{2}$ and

$$
\varepsilon= \begin{cases}-1 & \text { if }\left\{j_{1}, j_{2}\right\}=\{\sigma, \phi\}, \phi \in\left\{a_{1}, a_{3}\right\}, \\ 1 & \text { otherwise. }\end{cases}
$$

(2) the case $j_{1}=j_{2}=\sigma$

$$
\begin{gathered}
B\left[\begin{array}{ll}
\sigma & \sigma \\
\sigma & \sigma
\end{array}\right]=\frac{\gamma}{\sqrt{2}} \exp \frac{\pi \sqrt{-1}}{4}\left(\begin{array}{cc}
1 & -\sqrt{-1} \\
-\sqrt{-1} & 1
\end{array}\right), \\
B\left[\begin{array}{ll}
\sigma & \sigma \\
1 & 1
\end{array}\right]=B\left[\begin{array}{ll}
\sigma & \sigma \\
\psi & \psi
\end{array}\right]=\gamma, \quad B\left[\begin{array}{ll}
\sigma & \sigma \\
1 & \psi
\end{array}\right]=B\left[\begin{array}{ll}
\sigma & \sigma \\
\psi & 1
\end{array}\right]=\gamma \sqrt{-1} .
\end{gathered}
$$

Here $\gamma^{2}=\exp \left(-\frac{\pi \sqrt{-1}}{4}\right)$. 
(b') $F_{X_{j_{1} j_{2}}}$ is the inverse map of $F_{X_{j_{1} j_{2}}^{+}}$.

(c)

$$
F_{U_{j}}\left(e_{a_{1}}\right)= \begin{cases}e_{a_{1}} \otimes e_{a_{2}} \otimes e_{a_{1}} & \text { if } j \in\{1, \phi\}, \\ \alpha\left(e_{a_{1}} \otimes e_{\sigma} \otimes e_{a_{1}}\right) & \text { if } j=\sigma, a_{1} \in\{1, \phi\}, \\ \frac{\alpha}{\sqrt{2}}\left(e_{\sigma} \otimes e_{1} \otimes e_{\sigma}+e_{\sigma} \otimes e_{\psi} \otimes e_{\sigma}\right) & \text { if } j=a_{1}=\sigma .\end{cases}
$$

where $\alpha^{2}=\sqrt{2}$.

(d)

$$
F_{U \jmath}\left(e_{a_{1}} \otimes e_{a_{2}} \otimes e_{a_{1}}\right)= \begin{cases}e_{a_{1}} & \text { if } j \in\{1, \phi\}, \\ \alpha e_{a_{1}} & \text { if } j=\sigma, a_{1} \in\{1, \phi\}, \\ \frac{\alpha}{\sqrt{2}} e_{a_{1}} & \text { if } j=a_{1}=\sigma .\end{cases}
$$

We make a list of the above operators in Fig. 22 .

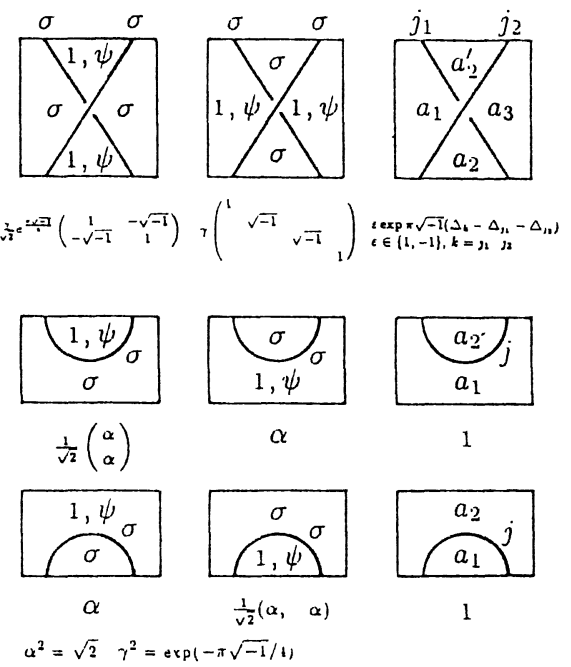

Fig. 22

A homomorphism for any link diagram is a composition of homomorphisms obtained by combining the operator for only one critical point with identity operators for strings passing from the top to the bottom without crossings. By the definition of the operators, for any link diagram $\Gamma(L)$, we obtain a homomorphism $F_{\Gamma(L)}: C \rightarrow C$. Thus $F_{\Gamma(L)}$ is a multiplication by some complex number $\mu$. We put $\tau_{L, \lambda}=\mu$.

Theorem 4.1. For any coloured framed link $L$ and any colouring map $\lambda$, $\tau_{L, \lambda}$ is invariant under regular isotopy for the diagram $\Gamma(L)$. Let $T_{j}$ be a dia- 
gram pictured in Fig. 23. The homomorphism $F_{T_{j}}$ is a multiplication by $\exp 2 \pi \sqrt{ }-1 \Delta_{\jmath}=v_{\jmath}$.

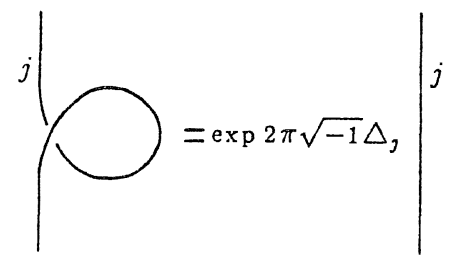

Fig. 23

Proof. By the definition of the operators $F_{X_{\jmath \jmath}^{+}}, F_{U_{\jmath}}, F_{U^{\jmath}}$, we can show that $F_{T_{j}}$ is a multiplication by the $\exp 2 \pi \sqrt{-1} \Delta_{j}$. To prove invariance under regular isotopy, it is enough to show that the homomorphisms defined by the isotopic diagrams in Fig. 24 are equal, for any colouring $\lambda([8],[11],[17])$. We can verify it by an easy calculation.
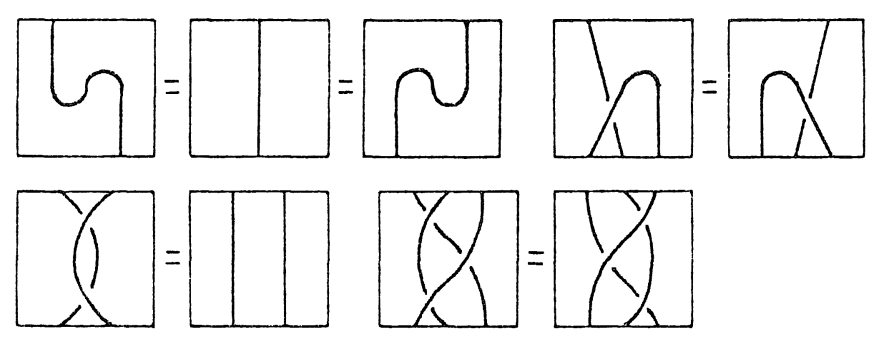

Fig. 24

We give some examples.

Example 4.2. Let $L$ be an unknotted circle with zero framing. If $\lambda(L)=i$, which is an element $i$ of the set $\{1, \sigma, \phi\}$, then we denote $\tau_{L, \lambda}$ by $\delta_{i}$.

Then we deduce : $\delta_{1}=\delta_{\omega}=1, \delta_{\sigma}=\sqrt{2}$.

Example 4.3. Let $H$ be the Hopf link which have zero framings. We assume that one component is assigned with $i$ and another with $j$. Then we write the invariant of $\Gamma(H)$ by $\widetilde{S}_{i j}$. Let us put

$$
S_{i,}=\frac{1}{2} \widetilde{S}_{i}
$$

and

We deduce

$$
S=\left(\begin{array}{lll}
S_{11} & S_{1 \sigma} & S_{1 \psi} \\
S_{\sigma 1} & S_{\sigma \sigma} & S_{\sigma \psi} \\
S_{\psi 1} & S_{\psi^{\prime} \sigma} & S_{\psi \psi}
\end{array}\right) .
$$




$$
S=\frac{1}{2}\left(\begin{array}{ccc}
1 & \sqrt{2} & 1 \\
\sqrt{2} & 0 & -\sqrt{2} \\
1 & -\sqrt{2} & 1
\end{array}\right) .
$$

In the same way as in [13], we construct invariants of 3-manifolds, using the above invariants of link diagrams.

Let $M$ be a closed connected oriented 3-manifold and $L$ a framed link with components $L_{1}, \cdots, L_{m}$ such that $M$ is homeomorphic to 3-manifold $M_{L}$ obtained by Dehn surgery of $S^{3}$ along $L$. We denote the set of colourings of $\Gamma(L)$ by $\operatorname{col}(L)$ and the signature of the linking matrix of $L$ by $\sigma(L)$. Then put

$$
\tau(M ; L)=C^{\sigma(L)} \sum_{\lambda \in \operatorname{col}(L)} \prod_{i=1}^{m} d_{\lambda\left(L_{i}\right) \tau_{L}, \lambda},
$$

where $C$ and $d_{i}$ for $i \subseteq\{1, \sigma, \phi\}$ are complex numbers characterized by

$$
\sum_{i \in\left(1, i, i_{i}^{\prime}\right)} C d_{i} \widetilde{S}_{i, \eta_{i}}=\eta_{,}^{-1} \delta_{l}
$$

for any $j \in\{1, \sigma, \phi\}$ and

$$
C=\sum_{i \in(1, \sigma, \psi)} v_{i}^{-1} \delta_{i} d_{i}
$$

Here $\delta_{i}$ is the same as in Example 4.2. More explicitly, we have

$$
\begin{gathered}
d_{1}=d_{\psi}=\frac{1}{2}, \quad d_{\sigma}=\frac{\sqrt{2}}{2}, \\
C=\sum_{i \in[1, \sigma, \psi)} v_{i}^{-1} \delta_{i} d_{i}=\exp \frac{-\pi \sqrt{-1}}{8} .
\end{gathered}
$$

Theorem 4.4. Let $M$ be a closed connected oriented 3-manifold and let $L$ be a framed link so that $M$ is the result of surgery along $L$ on $S^{3}$. Then $\tau(M ; L)$ is a topological invariant of $M$.

The proof of this theorem is similar to that of Theorem 1.6 (see [13]). To prove Theorem 4.4, we need the following lemma.

Lemma 4.5. Let $\Gamma_{1}(i, j), \Gamma_{2}(j)$ be the cloured $(1,1)$-tangles shown in Fig. 25.

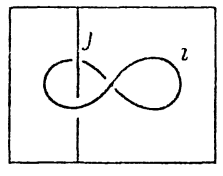

$\Gamma_{1}(i, \jmath)$

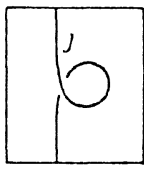

$\Gamma_{2}(\jmath)$

Fig. 25

Then 


$$
\sum_{i \in\{1, \sigma, \psi\}} C d_{i} \tau_{\Gamma_{1}(i, j)}=\tau_{\Gamma_{2}(j)} \in \operatorname{End}_{C}(V \otimes V)
$$

Proof. From the fact that $d_{i}$ satisfies the following equation (4.3)

$$
\sum_{i \in\{1, \sigma, \psi\}} C d_{i} \widetilde{S}_{i j} v_{i}=v_{j}^{-1} \delta_{j}
$$

we can obtain this lemma.

Let us introduce new tangle diagrams and operators corresponding to them. We consider tangle diagrams shown in Fig. 26. The corresponding operators are defined from fusing matrices.

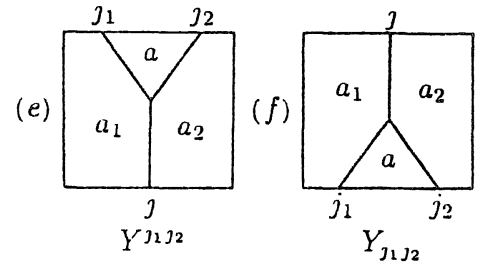

Fig. 26

In Fig. 26, $j$ is equal to 1 or $\phi$ if $j_{1}=j_{2}=\sigma$ and $j_{1} \cdot j_{2}$ otherwise.

(e)

In other cases,

$$
F_{Y^{\sigma} \sigma}\left(e_{\sigma} \otimes e_{\sigma}\right)=\sum_{a \in[1, \psi 1} F_{\jmath a}\left[\begin{array}{ll}
\sigma & \sigma \\
\sigma & \sigma
\end{array}\right]\left(e_{\sigma} \otimes e_{a} \otimes e_{\sigma}\right)
$$

$$
F_{Y^{j_{1} j_{2}}}\left(e_{a_{1}} \otimes e_{a_{2}}\right)=F_{j a}\left[\begin{array}{ll}
j_{1} & j_{2} \\
a_{1} & a_{2}
\end{array}\right]\left(e_{a_{1}} \otimes e_{a} \otimes e_{2}\right)
$$

where $a$ is uniquely determined from $a_{1}, a_{2}, j_{1}, j_{2}$.

(f) $F_{Y_{j_{1} j_{2}}}$ is a multiplication by a scalar.

$$
F_{Y_{j_{1} j_{2}}}\left(e_{a_{1}} \otimes e_{a} \otimes e_{a_{2}}\right)=F_{a j}\left[\begin{array}{cc}
a_{1} & a_{2} \\
j_{1} & j_{2}
\end{array}\right]\left(e_{a_{1}} \otimes e_{a_{2}}\right) .
$$

In terms of the above operators, we obtain the following lemma.

Lemma 4.6. Suppose $k \geqq 2$. Let $\Gamma_{i}, \tilde{\Gamma}_{i, p}, \Gamma_{2}$ and $\tilde{\Gamma}_{2, p}$ be tangle diagrams pictured in Fig. 27, where the indices present colours assigned to strings, i.e., elements of the $\{1, \sigma, \phi\}$. Then

$$
F_{\Gamma_{\imath}}= \begin{cases}F_{\tilde{\Gamma}_{i} p} & \text { if } p=j_{1} \cdot j_{2},\left(j_{1}, j_{2}\right) \neq(\boldsymbol{\sigma}, \boldsymbol{\sigma}) \\ \sum_{p=1, \psi} F_{\tilde{\Gamma}_{i, p}} & \text { if } j_{1}=j_{2}=\sigma,\end{cases}
$$




$$
F_{\Gamma^{\prime} 2}= \begin{cases}F_{\Gamma_{2}, p} & \text { if } p=j_{1} \cdot j_{2},\left(j_{1}, j_{2}\right) \neq(\sigma, \sigma) \\ \sum_{p=1, \psi} F_{\Gamma_{2}, p} & \text { if } j_{1}=j_{2}=\sigma .\end{cases}
$$

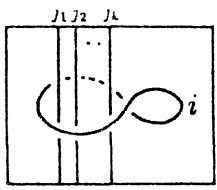

$\Gamma_{\imath}$

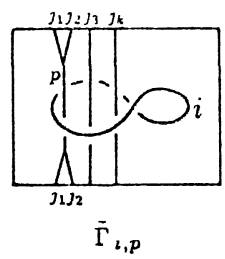

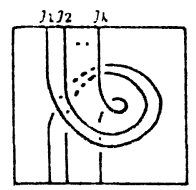

$\Gamma_{2}$

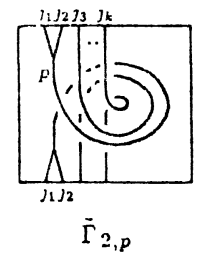

Fig. 27

Proof. The diagram $\tilde{\Gamma}_{i, p}$ is gained by applying the moves depicted in Fig. 28 to $\Gamma_{i}$.

Moreover, in addition to the above moves, we can obtain $\tilde{\Gamma}_{2, p}$ by applying the moves shown in Fig. 28 to $\Gamma_{2}$. Thus it is enough to prove simply invariance of the homomorphism under these moves.
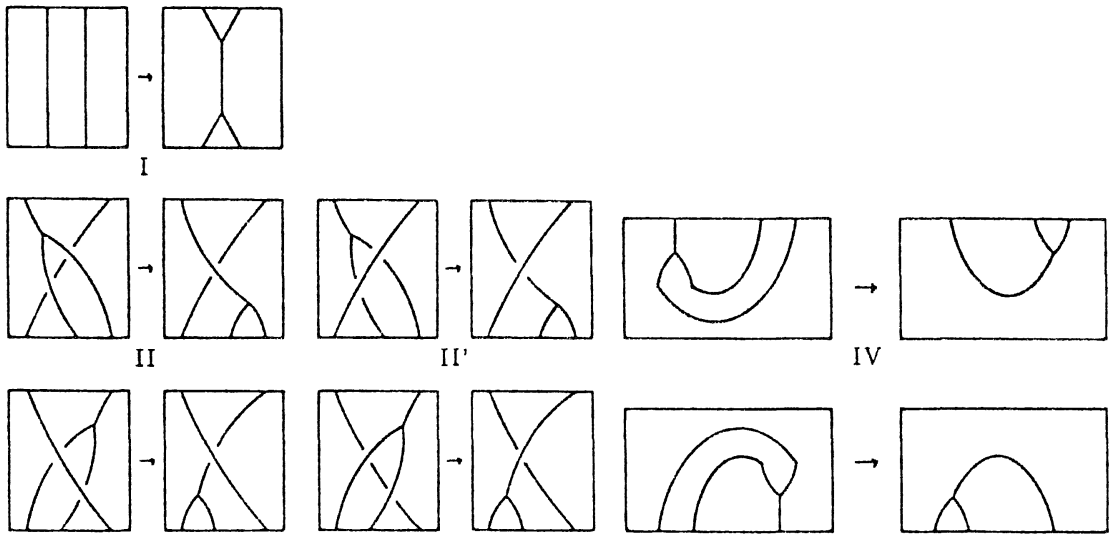

III
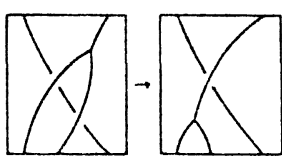

III

Fig. 28

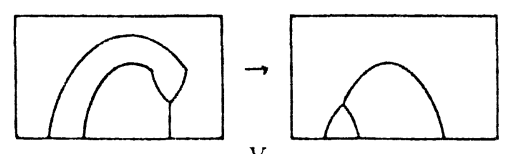

V

. 


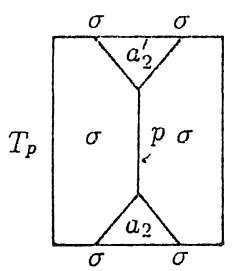

Fig. 29

tions for the homomorphisms $F_{T_{1}}: V_{1} \rightarrow V_{2}$ and $F_{T_{\psi}}: V_{1} \rightarrow V_{2}$ are

$$
F_{T_{1}}: \frac{1}{2}\left(\begin{array}{ll}
1 & 1 \\
1 & 1
\end{array}\right), \quad F_{T_{\varphi}}: \frac{1}{2}\left(\begin{array}{rr}
1 & -1 \\
-1 & 1
\end{array}\right)
$$

by the definition of the operators for diagrams $Y_{\sigma \sigma}{ }_{\sigma \sigma}$ and $Y^{\sigma \sigma}$.

Then the homomorphism $\sum_{p=1, \dot{\varphi}} F_{T_{p}}$ is the identity. Except for the above case, the invariance of the homomorphisms under move I is trivial.

In the case II, II', III and III', we need some more elementary calculations. For example, we consider the case pictured in Fig. 30.
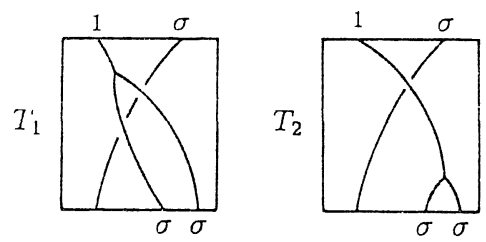

Fig. 30

Let $V_{1}$ be a vector space over $C$ with basis $\left\{e_{i_{1}} \otimes e_{\sigma} \otimes e_{i_{2}} \otimes e_{\sigma}\right\}_{i_{1}, i_{2}=1, \psi}$ and $V_{2}$ a vector space over $C$ with basis $\left\{e_{1} \otimes e_{1}, e_{\psi} \otimes e_{\psi}\right\}$. The restriction of $F_{T_{1}}$ and $F_{T_{2}}$ to $V_{1}$ determines $C$-linear homomorphisms $V_{1} \rightarrow V_{2}$.

The matrices for their are $\frac{1}{\sqrt{2}}\left(\begin{array}{llll}1 & 1 & 0 & 0 \\ 0 & 0 & 1 & 1\end{array}\right)$, one gets $\left.F_{T_{1}}\right|_{V_{1}}=\left.F_{T_{2}}\right|_{V_{2}}$. Similarly, comparing the matrix for $F_{T_{1}}$ with that for $F_{T_{2}}$ on the restriction of $F_{T_{1}}$ and $F_{T_{2}}$, we can prove that $F_{T_{1}}=F_{T_{2}}$.

Let us consider the cases IV and V. The definition of the operators in (e) and (f) implies that for any fusing matrix $F\left[\begin{array}{ll}a & d \\ b & c\end{array}\right]$,

$$
F\left[\begin{array}{ll}
a & d \\
b & c
\end{array}\right]=F\left[\begin{array}{ll}
d & c \\
a & b
\end{array}\right]
$$

Invariance of the homomorphisms in these cases follows from this. This completes the proof of the lemma.

Now we are in position to prove the theorem. 
Proof of Theorem 4.4. It suffices to verify that two Kirby moves on $L$ do not change $\tau(M ; L)$ (see $[13])$. The invariance under the first move, which is an elimination or insertion of an unknotted component with framing \pm 1 , can be derived from the definition of scalars $C$ and $d_{i}$. The second move is called Kirby $(+1)$-move, under which two diagrams are related as shown in the two above pictures in Fig. 27. Let us prove invariance under this move. It is enough to show the equation

$$
\sum_{i \in\{1, \sigma, \psi\}} C d_{i} F_{\Gamma_{i}}=F_{\Gamma_{2}} .
$$

We show it by induction with respect to $k$. When $k=1,(4.6)$ follows from Lemma 4.5. Suppose that it is true for $k=l$. We modify the diagram $\Gamma_{i}$ illustrated in Fig. 26 to $\tilde{\Gamma}_{i, p}$ in Fig. 26. The diagram $\tilde{\Gamma}_{i, p}$ may be regarded as the composition of three diagrams $\Gamma_{1}, \Gamma_{\imath}^{\prime}$, and $\Gamma_{3}$ illustrated in Fig. 31. Similarly, we modify the diagram $L_{2}$ in Fig. 31 to the diagram $\tilde{\Gamma}_{2, p}$. The diagram $\tilde{\Gamma}_{2, p}$ may be thought of as the composition of three diagrams $\Gamma_{2}, \Gamma_{2}^{\prime}, \Gamma_{3}$ in Fig. 31.

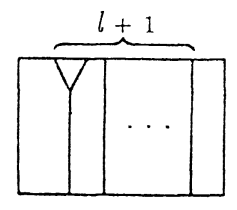

$\Gamma_{1}$

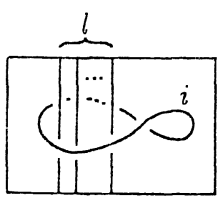

$\Gamma^{\prime \prime}$

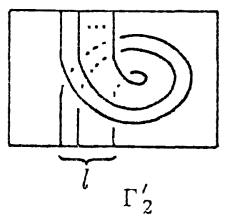

Fig. 31

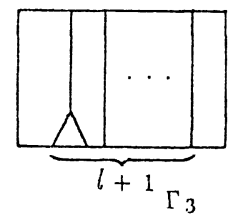

$\Gamma_{3}$

Then from the assumption of the induction, it follows that

$$
\sum_{\imath \in(1, \sigma, \phi)} d_{i} F_{\Gamma_{\imath}^{\prime}}=F_{\Gamma_{2}^{\prime}} .
$$

Thus

$$
\sum_{\imath \in(1, \sigma, \psi)} d_{i} F_{\Gamma_{i, p}}=F_{\Gamma_{2, p}} .
$$

Lemma 4.6 implies (4.6). This completes the proof.

We put $\tau(M ; L)=\tau(M)$.

Example 4.7. Since $S^{3}$ is obtained from an unknotted circle with framing 1,

$$
\tau\left(S^{3}\right)=C \sum_{i \in(1, \sigma, \psi)} d_{i} \delta_{i} v_{i}=1 .
$$

Since $S^{2} \times S^{1}$ is obtained from an unknotted circle with framing 0 , 


$$
\tau\left(S^{2} \times S^{1}\right)=\sum_{i \in(1, \sigma, \psi)} d_{i} \delta_{i}=2
$$

Proposition 4.8. The invariant $\tau(M)$ has the following properties.

(1) $\tau\left(M_{1} \# M_{2}\right)=\tau\left(M_{1}\right) \tau\left(M_{2}\right)$

(2) $\tau(-M)=\overline{\tau(M)}$, where ' $-M$ ' is a 3-manifold $M$ with reversed orientation and the bar is the complex conjugation.

Proof. For (1), let us $M_{L}$ be a 3-manifold obtained by Dehn surgery along a framed link $L$ in $S^{3}$. Choosing framed links $L_{1}$ and $L_{2}$ with $M_{L_{1}}=M_{1}, M_{L_{2}}$ $=M_{2}$, we obtain the equation $M_{L_{1} \cup L_{2}}=M_{1} \neq M_{2}$, where $L_{1} \cup L_{2}$ denotes disjoint union ( $L_{1}$ and $L_{2}$ are separated by a 2 -sphere), We note that for fixed colouring $\lambda_{i}$ of $L_{i}(i=1,2)$,

$$
\tau_{L_{1} \cup L_{2}, \lambda_{1} \cup \lambda_{2}}=\tau_{L_{1}, \lambda_{1}} \cdot \tau_{L_{2}, \lambda_{2}},
$$

where $\lambda_{1} \cup \lambda_{2}$ denotes the colouring of $L_{1} \cup L_{2}$ induced from the colourings $\lambda_{1}, \lambda_{2}$ of $L_{1}, L_{2}$. So (1) follows from the definition $\tau$.

For (2), one knows that $\left(-M_{L}\right)=M_{\bar{L}}$, while $\bar{L}$ is the mirror image of $L$. Since $\tau_{\bar{L}, \lambda}=\overline{\tau_{L, \lambda}}$ from the definition of homomorphisms in (b) and $\sigma(\bar{L})=-\sigma(L)$, one derives (2).

Remark 4.9. For the lens spaces $L(7,1)$ and $L(7,2)$,

$$
\tau(L(7,1))=\exp \frac{3}{4} \pi \sqrt{-1}, \quad \tau(L(7,2))=\exp \left(-\frac{\pi \sqrt{-1}}{4}\right) .
$$

Thus $\tau$ is not a homotopy invariant. In general, for the lens space $L(m, 1)$,

$$
\tau(L(m, 1))= \begin{cases}\exp \frac{(m-1) \pi \sqrt{-1}}{8} & \text { if } m \text { is odd, } \\ \exp \left(-\frac{\pi \sqrt{-1}}{8}\right)\left(1+\exp \frac{m \pi \sqrt{-1}}{8}\right) & \text { if } m \text { is even. }\end{cases}
$$

For the lens space $L(m, 2)$,

$$
\tau(L(m, 2))= \begin{cases}1 & \text { if } m=4 l+1, l=1,2, \cdots \\ \exp \left(-\frac{\pi \sqrt{-1}}{4}\right) & \text { if } m=4 l-1, l=1,2, \cdots\end{cases}
$$

Let $M$ be a closed connected oriented 3-manifold and $T$ a coloured $(0,0)$ tangle diagram in $M$. For a pair $(M, T)$, put

$$
\tau(M, T)=C^{\sigma(L)} \sum_{\lambda \in \operatorname{col}(L)} \prod_{i=1}^{m} d_{\lambda\left(L_{i}\right)} \tau_{\Gamma(L) \cup T, \lambda} .
$$

By the same discussion as in $\S 2$, we may have a projectively linear representation

$$
\rho: S L(2, Z) \longrightarrow G L\left(Z\left(T^{2}\right)\right) /\langle C\rangle,
$$


where corresponding matrices $S, T$ are defined by the formulas

$$
S=\frac{1}{2}\left(\begin{array}{ccc}
1 & \sqrt{2} & 1 \\
\sqrt{2} & 0 & -\sqrt{2} \\
1 & -\sqrt{2} & 1
\end{array}\right), \quad T=\left(\begin{array}{lll}
1 & \\
\exp \frac{\pi \sqrt{-1}}{8} & \\
& & -1
\end{array}\right) .
$$

We can also obtain 'Verlinde's formula' for this algebra.

\section{Acknowledgement}

I would like to thank Professor T. Kohno for useful advice. I also thank Professor E. Bannai for valuable remarks on a computation of 'Verlinde's formula'.

\section{References}

[1] Drinfel'd, V.G., Quantum groups, Amer. Math. Soc. (1987), 798-820.

[2] Ginsparg, P., Applied conformal Field Theory, Les Houches, Session XLIX, 1988, Champs, Cordes et Phénomènes Critiques/Fields, Strings and Critical Phenomena, ed. by E. Brézin and J. Zinn-Justin, Elsevier Science Publishers (1989).

[3] Jimbo, M., A $q$-difference analogue of $U(g)$ and the Yang-Baxter equation, Lett. Math. Phys., 10 (1985), 63-69.

[4] - Introduction to Yang-Baxter equation, Braid Group, Knot Theory and Statistical Mechanics, eds. C. N. Yang and M.L. Ge (World Scientific, 1989).

[5] Kac, V.G. and Peterson, D.H., Infinite dimensional Lie algebras, theta functions and modular forms, Advances in Math., 53 (1984), 125-264.

[6] Kirby, R., The calculus of framed links in $S^{3}$, Invent. Math., 45 (1978), 35-56.

[7] Kohno, T., Topological invariants for 3-manifolds using representation of mapping class groups $I$, to appear in Topology.

[8] Kirby, R. and Melvin, P., The 3-manifold invariants of Witten and ReshetikhinTuraev for sl(2,C), Invent. Math., 105 (1991), 473-545.

[9] Lickorish, W.B.R., A representation of orientable, combinatorial 3-manifolds, Ann. Math., 76 (1962), 531-540.

[10] Moore, G. and Seiberg, N., Classical and quantum conformal field theory, Commun. Math. Phys., 123 (1988), 177-254.

[11] Morton, S.R. and Strickland, P., Jones polynomial invariants for knots for knots and satellites, Math. Proc. Camb. Phil. Soc., 109 (1991), 83-103.

[12] Rolfsen, D., Knots and links, Boston Publish or Perish Press, 1976.

[13] Reshetikhin, N.Y. and Turaev, V.G., Invariants of 3-manifolds via link polynomials and quantum groups, Invent. Math., 103 (1991), 547-597.

[14] Reshetikhin, N.Y. and Turaev, V.G., Ribbon graphs and their invariants derived from quantum groups, Commun. Math. Phys., 127 (1990), 1-26.

[15] Verlinde, E., Fusion rules and modular transformations in $2 D$ conformal field theory, Nucl. Phys., B300 (1988), 360-376.

[16] Witten, E., Quantum field theory and the Jones polynomial, Commun. Math. Phys., 121 (1989), 351-399.

「17] Yetter, D. N., Markov algebra, Contemp. Math.. 78 (1988), 705-730, Braids, AMS. 
